\title{
El Mobbing en trabajadores universitarios: Una revisión sistemática
}

\section{Mobbing in University Workers: A Systematic Review}

\author{
José Luis Rojas-Solís* \\ Benemérita Universidad Autónoma de Puebla, Puebla, México \\ ORCID: http://orcid.org/0000-0001-6339-4607 \\ Brandon Enrique Bernardino García-Ramírez \\ Benemérita Universidad Autónoma de Puebla, Puebla, México \\ ORCID: http://orcid.org/0000-0001-9261-7427 \\ Manuel Edgardo Hernández-Corona \\ Benemérita Universidad Autónoma de Puebla, Puebla, México \\ ORCID: http://orcid.org/0000-0002-5449-6027
}

Recibido 30-07-19 Revisado 25-08-19 Aprobado 16-09-19 En línea 20-09-19

*Correspondencia

Email: jlrojassolis@gmail.com
Citar como:

Rojas-Solís, J., García-Ramírez, B., \& Hernández-Corona, M. (2019). El Mobbing en trabajadores universitarios: Una revisión sistemática. Propósitos y Representaciones, 7(3), 354-382. doi: http://dx.doi.org/10.20511/pyr2019.v7n3.369 


\section{Resumen}

Una gran variedad de estudios sobre acoso laboral han abordado diversos grupos laborales debido a que cada uno de ellos presenta condiciones organizacionales e individuales que facilita el desarrollo de esta problemática, por ello el presente trabajo realiza una revisión sistemática de investigaciones científicas sobre el acoso laboral en trabajadores universitarios, ocupando como herramienta de recolección las bases de datos; La Referencia, EBSCO, Web of Science, Scielo y SCOPUS considerando la información publicada en el periodo compuesto entre los años 2000 a 2019. Los estudios analizados fueron de enfoque cuantitativo y mixto, con diseño no experimental y transversal con un alcance correlacional y descriptivo. Con respecto a los instrumentos se resalta la importancia de la actualización teórica y de la validación, así como la necesidad de implementar y crear nuevos instrumentos para la medición de este fenómeno, del mismo modo se invita a realizar investigaciones del acoso laboral en trabajadores universitarios, considerando el análisis de diferencia por sexo y las conductas de acoso.

Palabras clave: Relaciones laborales; Relaciones entre pares; Universidad; Acoso; Revisión sistemática.

\section{Summary}

A great variety of studies on workplace harassment has been approached by different working groups because they show organizational and individual conditions that facilitate the development of this problem; for that reason the present study carries out a systematic review of scientific research on mobbing on university staff, using the following databases as a collection tool: La Referencia, EBSCO, Web of Science, Scielo and SCOPUS. The study included research published in the period between the years 2000 and 2019. The approach of the studies analyzed was quantitative and mixed, with a non-experimental and cross-sectional design, and a correlational and descriptive scope. With respect to the instruments used, the importance of theoretical updating and validation is highlighted, as well as the need to implement and create new instruments to measure this phenomenon. In the same way, research about mobbing on university staff in the workplace is encouraged, considering the analysis of sex differences and harassment behaviors.

Keywords: Labour Relations; Peer Relationship, Universities, Peer Harassment, Systematic Review.

\section{Introducción}

Las relaciones humanas están basadas en un conjunto de normas y comportamientos aceptados por la sociedad en la que se desarrollan. En ese orden de ideas se puede entender al ambiente laboral como el conjunto de características y propiedades (temporales o permanentes) que influyen en el comportamiento, desempeño y satisfacción laboral (Nares et al., 2014). En conjunto, el cumplimiento de estas condiciones facilita el desarrollo de un ambiente de trabajo saludable, lo cual, en proporción, aumenta la productividad y el bienestar de los empleados involucrados (Barrios \& Paravic, 2006). Es por ello que las relaciones sociales adquieren importancia dentro del espacio laboral, esto se debe a que constituyen la esfera de lo público, la cual se diferencia de otras, como la doméstica, debido a que sus miembros comparten objetivos y tareas en común (Tonon, 2012). Esta interacción interpersonal hace uso de un principio de comunicación el cual consiste en la transmisión de ideas consecuentes (Nares, García, Arvizu \& Olimón, 2014).

Así, un entorno laboral saludable favorece la productividad y la convivencia de los trabajadores, y, a su vez, mejora el bienestar de sus colaboradores y las relaciones interpersonales de estos (Barrios \& Paravic, 2006); mientras que el espacio de trabajo que no cumple con estas 
características favorece en los involucrados la acumulación de estrés y, posteriormente, consecuencias en la salud (Gómez, 2009; Mihyun, Sung-Hyun \& Hyun-ja, 2014).

El síntoma más común de un ambiente laboral dañino es la presencia de estrés, éste se puede entender como una respuesta ante un conjunto de estímulos hostiles, en la cual puede generar una actitud de hostigamiento o acoso (Gómez, 2009). De manera más concreta, el acoso tiene características que varían en función de los individuos involucrados en dicha relación y que dificulta el establecimiento de una definición precisa del ambiente (Bowling \& Beehr, 2006). De ese modo, un ambiente laboral dañino propicia el deterioro de las condiciones laborales que, generalmente, suelen expresarse como malestar psicológico, conductas de hostigamiento y/o manifestaciones psicosomáticas (Anjum, Ming, Siddiqi, \& Rasool, 2018; Gómez, 2009).

En relación con el ambiente universitario, la presencia de este fenómeno genera una decadencia en el desempeño laboral en el personal docente y administrativo (maestros, coordinadores, prefectos) y académico en lo que atañe a a los estudiantes (Balducci, Cecchin \& Fraccaroli, 2012; Bheenuck, Miers \& Pollard, 2007).

\section{Delimitación del fenómeno de acoso/mobbing}

El concepto de acoso laboral se define como todo comportamiento interpersonal cuya intención es la de agredir o perjudicar la integridad física o psicológica de cualquier empleado (Henning et al., 2017), aunque es preciso señalar que cada ambiente laboral determina las condiciones bajo las cuáles una acción se considera como acoso (Trujillo, Valderrabano \& Hernández, 2007).

El comportamiento hostil hacia los trabajadores es un fenómeno conocido como mobbing, término acuñado por Heinz Leymann a principios de 1980 (Romero-Pérez, 2006). No obstante, fueron Einarsen, Hoel, Zapf y Cooper (2003, p. 15) y que hace referencia al acto de acosar, ofender, excluir socialmente o afectar de manera negativa las tareas/trabajo de un individuo, convirtiéndolo en el objetivo, de forma sistemática, de actos sociales negativos.

Aunado a esto, no existe, hasta el momento de realizar la presente revisión, ninguna definición de violencia o acoso laboral unívocamente compartida entre los expertos pues en distintos países se utilizan una variedad de términos para aludir al mismo fenómeno (Tomei, 2018). Al igual que la definición general del acoso laboral, el término mobbing se encuentra sujeto a la contextualización del ambiente en el que se manifiesta, por lo que carece de una definición precisa (Trujillo et al., 2007); sin embargo, existe un conjunto de características que engloban esta conducta, las cuales son: ejercer un poder, jurídico y/o físico, mediante una fuerza intimidatoria, perjudicar la integridad física y/o mental, su intención es la de denigrar u ofender a la víctima y, en mayor medida, este conjunto de conductas deteriora el ambiente laboral (RomeroPérez, 2006).

El criterio para considerar a la agresión como mobbing es que debe ocurrir de manera repetida y regular durante un periodo de tiempo prolongado, en el que uno solo domina en el acto (Einarsen et al., 2003). Contrario a lo que se piensa, el acoso laboral no es ejercido por un solo tipo de poder (Henning et al., 2017). Así es entendible que solo se dispongan de generalizaciones empíricas al respecto del fenómeno, es decir proposiciones de distintos autores que han sido comprobadas en la mayor parte de las investigaciones realizadas (Hernández, Fernández \& Baptista, 2014).

\section{Incidencia del acoso/mobbing}

Las manifestaciones del mobbing varían con respecto a la disposición de las condiciones laborales, así mismo las conductas más comunes abarcan un abanico de conductas que pueden ser; gritos, discriminación, insultos directos, señales y miradas obscenas, y en los casos de mayor intensidad, agresiones físicas graves; en casos extremos se puede producir un homicidio (Bowling 
\& Beehr, 2006). La incidencia de este fenómeno, al igual que sus consecuencias, está ligada a diferentes factores (Einarsen \& Hauge, 2006).

Estudios revelan que la práctica de acoso más común es de carácter sexual, esto involucra toda conducta inapropiada que involucre hostigamiento y comportamiento hostil (Asquith, Ferfolia, Brady \& Hanckel, 2018; Marsh et al., 2009) que atente contra la integridad sexual de la víctima (Eaton, 2004; O’Hare \& O’Donohue,1998; Ohse \& Stockdale, 2008).

Respecto al acoso dentro del personal universitario, se entiende que toda institución educativa representa un espacio en el que las interacciones influyen en el desempeño laboral y el bienestar de los involucrados (Moreno, Sepúlveda \& Restrepo, 2012). El desarrollo de relaciones saludables promueve la productividad en el ambiente escolar para funcionarios, personal y estudiantes (López-Cabarcos, Picón-Prado \& Vázquez-Rodríguez, 2008; Nares et al., 2014). En este caso, las incidencias de acoso laboral no presentan una diferencia significativa en comparación a otras instituciones (Henning et al., 2017).

La investigación hecha por Parra \& Acosta (2010) concluye que el contexto de trabajo en la universidad presenta condiciones individuales y organizacionales que favorecen el posible desarrollo del acoso psicológico y el mismo no se encuentra limitado a grupos particulares ya que afecta a todos por igual (Piñuel, 2001).

En Latinoamérica se estima que la prevalencia de acoso laboral se encuentra entre el 3,5\% y el 25,37\% (Pando, Aranda \& Olivares, 2012), mientras que la Cuarta Encuesta Europea Sobre las Condiciones del Trabajo aplicada en 2005 y realizada por la Fundación Europea para la Mejora de las Condiciones de Vida y Trabajo (2006) indica que la prevalencia en el sector europeo se encuentra en el $5 \%$, pero se estima que los porcentajes podrían superar la media y oscilar entre el $2 \%$ y el $17 \%$.

\section{Consecuencias del acoso/mobbing}

El acoso laboral puede generar alteraciones físicas como sensación de ahogo, palpitaciones, cansancio, pánico, inseguridad, desconfianza, dejadez, pérdida memoria o hipervigilancia, siendo las repercusiones psicológicas las más frecuentes (Trujillo et al., 2007). Así mismo, el comportamiento hostil hacia los trabajadores aumenta los niveles de estrés mientras que produce una decadencia en el desempeño laboral y bienestar emocional de las víctimas (Rospenda, Richman, Ehmke, \& Zlatoper, 2005). La consecuencia psicológica más común es la presencia de un episodio depresivo mayor o, si es regular y constante, el estado de distimia (Nares et al., 2014; Rospenda et al., 2005).

En conjunto con esto, la víctima sufre cambios significativos en su personalidad, esto debido a la pérdida gradual de confianza y la autoestima que este estado provoca (Nares et al., 2014; Rospenda et al., 2005; Trujillo et al., 2007).

\section{Método}

En consecuencia, el presente trabajo pretende revisar los estudios realizados con respecto a la vinculación entre acoso laboral (mobbing) y sus efectos en el personal universitario. Se utilizó la literatura encontrada en las bases de datos, La referencia, Web of Science, Scielo, Scopus y, en un caso especial, EBSCO, para ello se utilizaron métodos sistemáticos y explícitos para localizar, seleccionar y valorar críticamente las investigaciones relevantes, según las orientaciones derivadas de la Declaración PRISMA (Hutton, Catalá-López, \& Moher, 2016).

El objetivo general explorar el estado de la cuestión con respecto al acoso laboral en el personal universitario, específicamente se busca describir la prevalencia y las principales características metodológicas de los estudios realizados sobre la materia en el periodo de 
recolección desde el año 2000 a junio de 2019. Así, se pretende identificar la incidencia/prevalencia y principal tipo de acoso dentro del personal universitario, así como la identificación de los principales aspectos metodológicos de las investigaciones. Se estableció como objetivo secundario identificar las principales características (ubicación temporal y validación) de los instrumentos utilizados para las investigaciones realizadas que cumplen con los criterios de inclusión de la presente revisión.

\section{Selección de artículos}

La búsqueda fue realizada con los términos "workplace harrasment" y "university staff" para la exploración en el idioma inglés, mientras que en castellano se utilizaron los términos "acoso laboral" y "personal universitario". Con el fin de garantizar la exhaustividad del estudio, se creó la cadena de búsqueda agregando el operador boleano AND y los términos previamente mencionados. Como resultado de esto, la cadena de búsqueda fue "Workplace harrasment AND "University staff". Durante el proceso de búsqueda el protocolo de investigación se llevó a cabo en varias ocasiones a través de diferentes redes de área local con acceso a Internet para garantizar la reproducibilidad de la revisión.

El ingreso a las bases de datos correspondientes fue posible gracias al acceso otorgado por parte de la Benemérita Universidad Autónoma de Puebla. En caso del sitio EBSCO, se realizó una búsqueda avanzada usando los términos "workplace harassment" AND "university staff", se marcó la opción de "buscar todos mis términos de búsqueda", seguido de seleccionar las bases de datos Academic Search Complete, eBook Collertion (EBSChost), MedicLatina, Newswires, CINAHL with Full Text, Dentistry \& Oral Sciences Sources, Fuente Académica Premier, MEDLINE Complete y SPORTDiscus with Full Text; se limitaron los resultados a texto completo, en un periodo de publicación del año 2000 al 2019, y en idiomas inglés y español. El periodo de búsqueda y recolección de datos se realizó durante los meses de marzo a mayo del año 2019.

\section{Criterios de inclusión}

Los criterios técnicos de inclusión fueron: tener las características de un artículo empírico y arbitrado, se descartaron las revisiones, capítulos de libro y tesis de maestría. La investigación fue complementada con los filtros de búsqueda proporcionados por los sitios en línea de las bases de datos, los cuales fueron: año de publicación (2000-2019), texto completo e idioma (español e inglés). Así mismo, los artículos incluidos tienen términos y factores asociados al acoso laboral en el personal universitario.

\section{Proceso de selección}

Como resultado de la búsqueda se encontraron 2 resultados en idioma español y 69 en inglés para las bases La referencia, Web of Science/ colección completa, Scielo y Scopus. En caso de la base EBSCO los resultados fueron 59 en inglés y 6 en español, sin embargo, debido al algoritmo automático de esta base de datos, se redujeron a 44 en idioma inglés y 3 en español. Esto da como resultado un total de 71 artículos para las primeras bases de datos y 47 para EBSCO, sumando un total de 118 artículos, de las cuales fueron eliminados 110 debido a la aplicación de los criterios mencionados (véase Figura 1).

\section{Información extraída}

El análisis de las investigaciones incluidas contempla la ubicación temporal de la publicación, la propia metodología emprendida, los instrumentos utilizados, factores asociados y de riesgo, hallazgos, limitaciones, consideraciones éticas, prevalencia e incidencia. 
El proceso de búsqueda, identificación y selección de los estudios se realizó en cuatro fases (Véase Figura 1):

\section{Fase 1}

Se recolectaron los datos generales de los 118 estudios encontrados por medio de los criterios de búsqueda. La información obtenida de este proceso fue el título del estudio, nombre del autor, fecha y país de publicación, tipo de publicación, DOI y la base de datos en la que se encontraron. Además de haber identificado los estudios duplicados, así como los que carecieron de acceso abierto.

\section{Fase 2}

Se identificaron los estudios con acceso al texto completo por medio de la base de datos correspondiente, así como los que se obtuvieron a través de una fuente externa.

\section{Fase 3}

Se descartaron 55 artículos que no cumplieron con los criterios mencionados en la Fase 1 y 2 . Se obtuvieron 63 estudios, de los cuales se señalaron 8 como candidatos a lectura tomando como principal criterio que la muestra fuera parte del personal universitario.

\section{Fase 4}

Se realizó el análisis de lectura de los 8 artículos seleccionados en la Fase 3. Se identificaron los hallazgos, factores de riesgo, factores protectores, limitaciones, las futuras líneas de investigación, las consideraciones éticas y la prevalencia/incidencia de cada estudio.

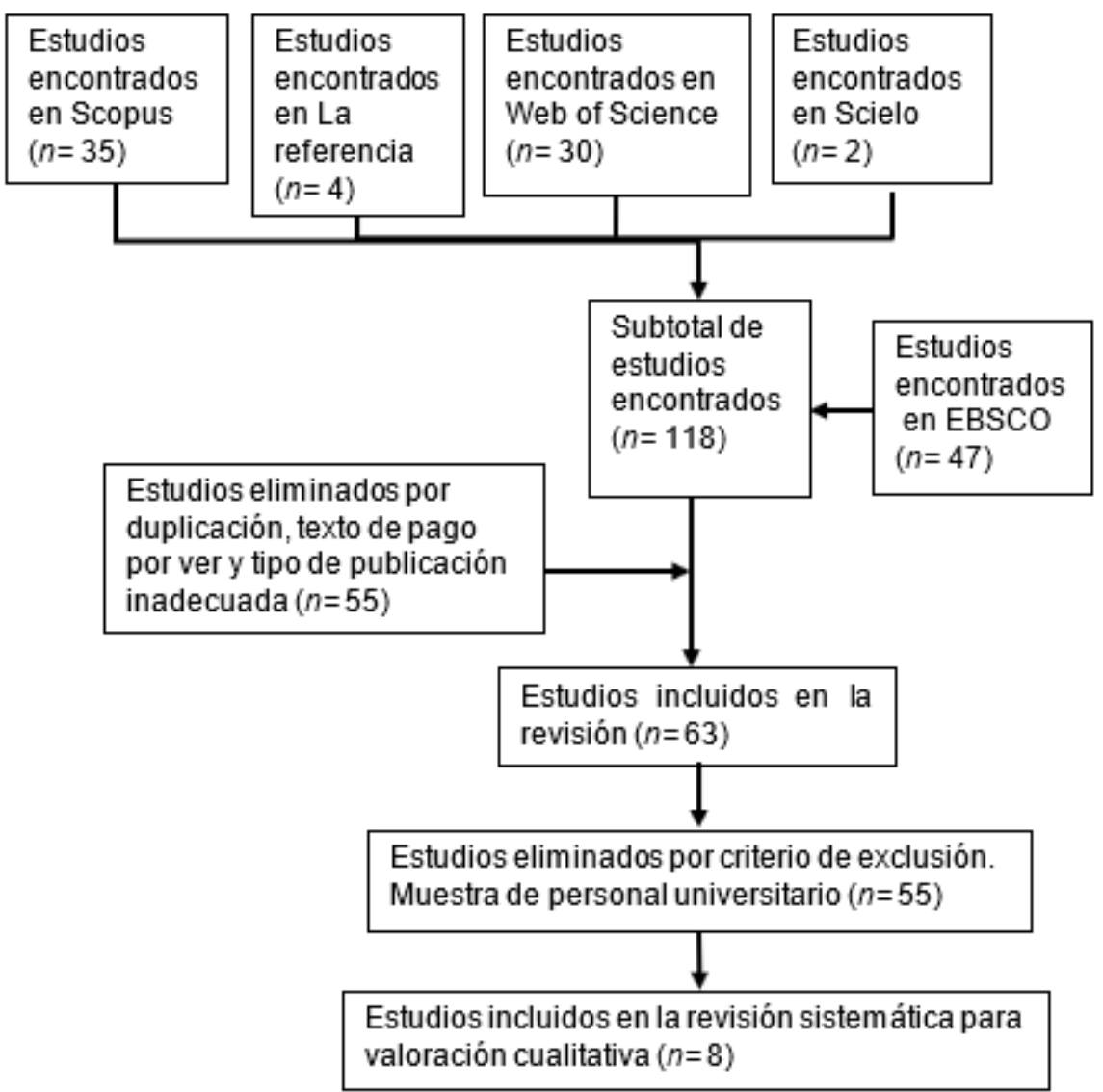

Figura 1. Diagrama PRISMA (Fuente: Elaboración propia) 


\section{Resultados}

En la Tabla 1 puede apreciarse a partir de los trabajos empíricos seleccionados para análisis, con lo que respecta el periodo 2000-2004 se no se hallaron artículos, el periodo 2005-2009 se concentra un $37.5 \%$ de publicaciones realizadas, $12.5 \%$ en $2010-2014$ y $50 \%$ en $2015-2019$. Esto demuestra que la aportación de conocimiento científico, con respecto al estudio empírico, en la violencia en el trabajo es bastante escaso en América Latina (Ansoleaga, Gómez-Rubio \& Mauro, 2015).

En cuestión de la metodología utilizada por parte de los estudios analizados (véase Tabla 3 ), se puede apreciar que el enfoque más utilizado es el cuantitativo (87.5\%) mientras que uno sólo (12.5\%) utilizó un enfoque mixto. Todos los estudios incluidos son de diseño transversal. En lo que concierne al alcance del estudio, dos de los trabajos (25\%) son descriptivos, mientras que cinco $(62.5 \%)$ son no experimentales/correlacionales. Por otra parte, existe un caso de alcance no experimental/descriptivo (12.5\%).

Tabla 1.

Principales aspectos de los estudios analizados

\begin{tabular}{|c|c|c|c|}
\hline Autor(es) y Año & $\begin{array}{c}\text { Enfoque / Diseño } \\
\text { / Alcance }\end{array}$ & Muestra & Procedimientos éticos \\
\hline $\begin{array}{l}\text { Marsh et al., } \\
2009\end{array}$ & $\begin{array}{l}\text { Cuantitativo / No } \\
\text { experimental, } \\
\text { transversal/ } \\
\text { Descriptivo }\end{array}$ & $\begin{array}{l}\text { Seleccionada de } \\
\text { manera aleatoria y } \\
\text { significativa }\end{array}$ & $\begin{array}{l}\text { Hubo consentimiento } \\
\text { informado } \\
\text { Se aseguró anonimato, } \\
\text { voluntariedad y } \\
\text { confidencialidad } \\
\text { Hubo comité de ética }\end{array}$ \\
\hline $\begin{array}{l}\text { Coskuner et al., } \\
2017\end{array}$ & $\begin{array}{l}\text { Cuantitativo/ No } \\
\text { experimental, } \\
\text { transversal / } \\
\text { Correlacional }\end{array}$ & $\begin{array}{l}\text { Seleccionada por } \\
\text { conveniencia }\end{array}$ & $\begin{array}{l}\text { Hubo consentimiento } \\
\text { informado } \\
\text { Se aseguró anonimato, } \\
\text { voluntariedad y } \\
\text { confidencialidad }\end{array}$ \\
\hline $\begin{array}{l}\text { Merilainen, } \\
\text { Sinkkonen, Puha } \\
\text { kka, y Kayhko, } \\
2016\end{array}$ & $\begin{array}{l}\text { Cuantitativo / No } \\
\text { experimental, } \\
\text { transversal / } \\
\text { Descriptivo }\end{array}$ & $\begin{array}{l}\text { Seleccionada por } \\
\text { conveniencia }\end{array}$ & $\begin{array}{l}\text { Se aseguró anonimato, } \\
\text { voluntariedad y } \\
\text { confidencialidad }\end{array}$ \\
\hline $\begin{array}{l}\text { Skinner et al., } \\
2015\end{array}$ & $\begin{array}{l}\text { Cuantitativo/ No } \\
\text { experimental, } \\
\text { transversal / } \\
\text { Correlacional }\end{array}$ & $\begin{array}{l}\text { Seleccionada de } \\
\text { manera aleatoria y } \\
\text { significativa }\end{array}$ & $\begin{array}{l}\text { Hubo consentimiento } \\
\text { informado } \\
\text { Se aseguró anonimato, } \\
\text { voluntariedad y } \\
\text { confidencialidad } \\
\text { Hubo comité de ética }\end{array}$ \\
\hline $\begin{array}{l}\text { Kang y Sidhu, } \\
2015\end{array}$ & $\begin{array}{l}\text { Cuantitativo / No } \\
\text { experimental, } \\
\text { transversal / } \\
\text { Correlacional }\end{array}$ & $\begin{array}{l}\text { Seleccionada por } \\
\text { conveniencia }\end{array}$ & $\begin{array}{l}\text { Se aseguró anonimato, } \\
\text { voluntariedad y } \\
\text { confidencialidad }\end{array}$ \\
\hline $\begin{array}{l}\text { Moreno, } \\
\text { Sepúlveda y } \\
\text { Restrepo, 2012 }\end{array}$ & $\begin{array}{l}\text { Mixto / No } \\
\text { experimental, } \\
\text { transversal / } \\
\text { Descriptivo }\end{array}$ & $\begin{array}{l}\text { Seleccionada por } \\
\text { conveniencia }\end{array}$ & $\begin{array}{l}\text { Hubo consentimiento } \\
\text { informado } \\
\text { Se aseguró anonimato, } \\
\text { voluntariedad y } \\
\text { confidencialidad }\end{array}$ \\
\hline $\begin{array}{l}\text { López-Cabarcos } \\
\text { et al., } 2008\end{array}$ & $\begin{array}{l}\text { Cuantitativo / No } \\
\text { experimental, } \\
\text { transversal / } \\
\text { Descriptivo }\end{array}$ & $\begin{array}{l}\text { Seleccionada por } \\
\text { conveniencia }\end{array}$ & $\begin{array}{l}\text { Se aseguró anonimato, } \\
\text { voluntariedad y } \\
\text { confidencialidad }\end{array}$ \\
\hline $\begin{array}{l}\text { Rospenda et al., } \\
2005\end{array}$ & $\begin{array}{l}\text { Cuantitativo / No } \\
\text { experimental, } \\
\text { transversal / } \\
\text { Correlacional }\end{array}$ & $\begin{array}{l}\text { Seleccionada por } \\
\text { conveniencia }\end{array}$ & $\begin{array}{l}\text { Hubo consentimiento } \\
\text { informado } \\
\text { Se aseguró anonimato, } \\
\text { voluntariedad y } \\
\text { confidencialidad } \\
\text { Hubo compensación } \\
\text { económica }\end{array}$ \\
\hline
\end{tabular}


Con respecto a la ubicación geográfica (véase Tabla 2) se puede apreciar que los estudios empíricos incluidos en el análisis del periodo 2000-2019 manifiesta una concentración geográfica en el continente americano (25\%), africano (12.5\%), europeo (25\%), oceánico (12.5\%), asiático (12.5\%) y europeo/asiático (12.5\%); esto refleja que la aportación del conocimiento respecto a este tema proceda sea principalmente del continente europeo.

\begin{tabular}{llll}
\hline País de la muestra & Continente & Tamaño de la muestra & $\begin{array}{l}\text { Prevalencia } \\
\text { /incidencia }\end{array}$ \\
\hline Etiopía & África & 387 & $54 \%$ \\
Colombia & América & 398 & $9.60 \%$ \\
Estados unidos & América & 2,492 & $12 \%$ \\
España & Europa & 321 & $8.70 \%$ \\
Finlandia & Europa & 114 & $45 \%$ \\
Turquía & Europa/Asia & 152 & $30 \%$ \\
India & Asia & 570 & $11.60 \%$ \\
Australia & Oceanía & 250 & $31 \%$ \\
\hline
\end{tabular}

Cuatro de los estudios (50\%) modificaron una escala original o construyeron una fundamentada en una o varias teorías. Por otra parte, una de las investigaciones (12.5\%) tiene un instrumento mixto y no especificado, mientras que el resto $(37.5 \%)$ utilizaron una escala sin modificar (véase Tabla 3).

El acoso laboral existe en el ámbito universitario y la manifestación más frecuente es de carácter sexual. Los estudios incluidos en el análisis han utilizado estudios transversales, de enfoque cuantitativo, descriptivo y correlacional.

La investigación encontró que no se han realizado estudios en México, hasta el momento de la realización de eta revisión sistemática. 
Tabla 3.

Instrumentos utilizados en los estudios incluidos en el análisis

\begin{tabular}{|c|c|c|c|}
\hline Autor(es) y Año & Instrumento(s) & Año & Validación posterior \\
\hline $\begin{array}{l}\text { Marsh et al., } \\
2009\end{array}$ & $\begin{array}{l}\text { Generalized Workplace } \\
\text { Abuse }\end{array}$ & 1999 & Validado por Richman et al. \\
\hline $\begin{array}{l}\text { Coskuner et al., } \\
2017\end{array}$ & $\begin{array}{l}\text { Leymann inventory of } \\
\text { psychological terror. } \\
\text { Perceived organizational } \\
\text { support. } \\
\text { Organizational } \\
\text { identification scale. } \\
\text { (Leymann) }\end{array}$ & $\begin{array}{r}1996 \\
1986 \\
1998\end{array}$ & $\begin{array}{l}\text { Para Turquía, por Erenler en } 2010 \\
\text { Para Turquia, por Erenler en } 2010 \\
\text { Para Turquía, por Güleryüz en } \\
2004\end{array}$ \\
\hline $\begin{array}{l}\text { Merilainen, } \\
\text { Sinkkonen, Puhakk } \\
\text { a, y Kayhko, } 2016\end{array}$ & $\begin{array}{l}\text { Negative Acts } \\
\text { Questionnaire-Revised } \\
\text { (NAQ-R) }\end{array}$ & 2009 & Validado por Einarsen et al. \\
\hline Skinner et al., 2015 & $\begin{array}{l}\text { Reported Harassment and } \\
\text { Serious Harassment by } \\
\text { Staff and University Type }\end{array}$ & 2012 & Validado por Strachan et al. \\
\hline Kang y Sidhu, 2015 & $\begin{array}{l}\text { Construcción de escala } \\
\text { propia a partir de: } \\
\text { The general health scale } \\
\text { An organizacional stress } \\
\text { screening (ASSET) } \\
\text { The Faculty Stress Index } \\
\text { (FSI) } \\
\text { Scale on stress }\end{array}$ & $\begin{array}{l}1978 \\
2002 \\
\\
1993 \\
2003\end{array}$ & $\begin{array}{l}\text { Validado por Goldberg } \\
\text { Validado por Cartwright \& } \\
\text { Cooper } \\
\text { Validado por Gmelch } \\
\text { Validado por Kinman y Jones }\end{array}$ \\
\hline $\begin{array}{l}\text { Moreno, Sepúlveda } \\
\text { y Restrepo, } 2012\end{array}$ & Mixto no especificado & - & - \\
\hline $\begin{array}{l}\text { López-Cabarcos et } \\
\text { al., } 2008\end{array}$ & $\begin{array}{l}\text { Construcción de escala } \\
\text { propia a partir de: } \\
\text { Definición de Mobbing } \\
\text { LIPT-60 } \\
\text { Consecuencias sobre la } \\
\text { salud } \\
\text { Respuesta de la víctima }\end{array}$ & $\begin{array}{l}1996 \\
2003 \\
\\
2001 \\
2003\end{array}$ & $\begin{array}{l}\text { Validado por Leyman } \\
\text { Validado por Gónzalez y } \\
\text { Rodríguez } \\
\text { Validado por Lazzarri } \\
\text { Validado por Piñuel \& Oñate }\end{array}$ \\
\hline $\begin{array}{l}\text { Rospenda et al., } \\
2005\end{array}$ & $\begin{array}{l}\text { Construcción de escala } \\
\text { propia a partir de: } \\
\text { Sexual experiences } \\
\text { questionnaire } \\
\text { The generalized workplace } \\
\text { harasment questionnaire } \\
\text { The job content } \\
\text { questionnaire } \\
\end{array}$ & $\begin{array}{l}1990 \\
2004 \\
1990\end{array}$ & $\begin{array}{l}\text { Validado por Fitzgerald } \\
\text { Validado por Rospenda \& } \\
\text { Richman } \\
\text { Validado por Karasek \& Theorell }\end{array}$ \\
\hline
\end{tabular}

En cuanto a los factores asociados, se encontró a los aspectos socioculturales como la principal influencia del acoso laboral (véase Tabla 4). Así mismo, los factores de riesgo que conlleva su incidencia están mayormente orientados a un desequilibrio general en el ambiente laboral, lo cual produce una decadencia en el desempeño de los involucrados e involucra factores socioculturales, educativos, sexuales y laborales (Einarsen et al., 2003). 
En promedio, la incidencia del fenómeno es de $25.23 \%$ del total de los estudios analizados. Un cuarto de la población incluida en la investigación es víctima de acoso laboral. Se identifica al acoso sociocultural como la principal manifestación.

A su vez, se le otorga el carácter discriminatorio al $75 \%$ del total de los estudios. Aunado a esto, se percibe un deterioro en las condiciones laborales, estado de salud física y mental de las víctimas. Así mismo, dicha sintomatología varía en relación con las condiciones en las que se desarrolla el acoso, sin embargo, se identifica al episodio depresivo mayor como consecuencia principal.

Tabla 4.

Factores identificados en los estudios analizados

\begin{tabular}{|c|c|c|c|c|}
\hline $\begin{array}{c}\text { Autor (es) y } \\
\text { Año }\end{array}$ & Influencias & $\begin{array}{l}\text { Factores de } \\
\text { riesgo }\end{array}$ & $\begin{array}{c}\text { Principal acoso } \\
\text { percibido }\end{array}$ & Consecuencias \\
\hline $\begin{array}{l}\text { Marsh et al., } \\
2009\end{array}$ & $\begin{array}{l}\text { Nivel educativo, } \\
\text { afiliación } \\
\text { religiosa y } \\
\text { socioculturales }\end{array}$ & $\begin{array}{l}\text { Acoso laboral y } \\
\text { acoso sexual. }\end{array}$ & Sociocultural & $\begin{array}{l}\text { Sintomatología de } \\
\text { depresión, } \\
\text { alteraciones en el } \\
\text { estado de ánimo, } \\
\text { riesgos a la salud } \\
\text { mental }\end{array}$ \\
\hline $\begin{array}{l}\text { Coskuner et al., } \\
2017\end{array}$ & Socioculturales & $\begin{array}{l}\text { Relación entre la } \\
\text { identidad social } \\
\text { y la jerarquia, las } \\
\text { oportunidades } \\
\text { de competencia } \\
\text { y mejores } \\
\text { salarios. }\end{array}$ & $\begin{array}{l}\text { Sociocultural } \\
\text { Discriminatorio }\end{array}$ & $\begin{array}{l}\text { Decadencia del } \\
\text { ambiente } \\
\text { organizacional y } \\
\text { educativo. } \\
\text { Competencia } \\
\text { entre miembros de } \\
\text { una institución }\end{array}$ \\
\hline $\begin{array}{l}\text { Merilainen, } \\
\text { Sinkkonen, } \\
\text { Puhakka, y } \\
\text { Kayhko, 2016 }\end{array}$ & $\begin{array}{l}\text { Socioculturales, } \\
\text { económicos y de } \\
\text { género }\end{array}$ & $\begin{array}{l}\text { Falta de } \\
\text { liderazgo, } \\
\text { sistemas de } \\
\text { competencia } \\
\text { (recompensas), } \\
\text { estatus. Estilos } \\
\text { de liderazgo y } \\
\text { gestión de } \\
\text { laissez-faire }\end{array}$ & $\begin{array}{l}\text { Sociocultural } \\
\text { Discriminatorio }\end{array}$ & $\begin{array}{l}\text { Efectos } \\
\text { psicosomáticos en } \\
\text { las víctimas del } \\
\text { acoso como el } \\
\text { síndrome de estrés } \\
\text { post-traumático } \\
\text { Declive del } \\
\text { entorno laboral y } \\
\text { reducción en la } \\
\text { rentabilidad de la } \\
\text { facultad }\end{array}$ \\
\hline $\begin{array}{l}\text { Skinner et al., } \\
2015\end{array}$ & $\begin{array}{l}\text { Socioculturales } \\
\text { y económicos }\end{array}$ & $\begin{array}{l}\text { Establecer } \\
\text { relaciones } \\
\text { jerárquicas, } \\
\text { Variación } \\
\text { regional del } \\
\text { idioma inglés }\end{array}$ & $\begin{array}{l}\text { Sociocultural } \\
\text { Discriminatorio }\end{array}$ & $\begin{array}{l}\text { Decadencia en las } \\
\text { relaciones } \\
\text { interpersonales y } \\
\text { desarrollo } \\
\text { profesional }\end{array}$ \\
\hline $\begin{array}{l}\text { Kang y Sidhu, } \\
2015\end{array}$ & $\begin{array}{l}\text { Nivel educativo } \\
\text { y socioculturales }\end{array}$ & $\begin{array}{l}\text { Desequilibrio } \\
\text { entre el trabajo y } \\
\text { la vida, } \\
\text { Comportamient } \\
\text { o } \\
\text { discriminatorio }\end{array}$ & $\begin{array}{l}\text { Sociocultural } \\
\text { Discriminatorio } \\
\text { De género }\end{array}$ & $\begin{array}{l}\text { Deterioro } \\
\text { desempeño } \\
\text { laboral }\end{array}$ \\
\hline $\begin{array}{l}\text { Moreno, } \\
\text { Sepúlveda y } \\
\text { Restrepo, } 2012\end{array}$ & $\begin{array}{l}\text { Socioculturales } \\
\text { y económicos }\end{array}$ & $\begin{array}{l}\text { Desequilibrio } \\
\text { entre el trabajo y } \\
\text { la vida, } \\
\text { Comportamient } \\
\text { o discriminatorio }\end{array}$ & $\begin{array}{l}\text { Sociocultural } \\
\text { Discriminatorio }\end{array}$ & $\begin{array}{l}\text { Decadencia en las } \\
\text { relaciones } \\
\text { interpersonales y } \\
\text { desarrollo } \\
\text { profesional }\end{array}$ \\
\hline $\begin{array}{l}\text { López-Cabarcos } \\
\text { et al., } 2008\end{array}$ & $\begin{array}{l}\text { Socioculturales } \\
\text { y económicos }\end{array}$ & $\begin{array}{l}\text { Las víctimas } \\
\text { señalan como } \\
\text { principales } \\
\text { consecuencias } \\
\text { del } \\
\text { mobbing/ } \\
\text { aspectos res con } \\
\text { relacionados salud } \\
\text { la psicológica y } \\
\text { emocional. }\end{array}$ & $\begin{array}{l}\text { Sociocultural } \\
\text { Discriminatorio }\end{array}$ & $\begin{array}{lr}\text { Deterioro } & \text { del } \\
\text { clima } & \text { laboral, } \\
\text { aspectos } & \\
\text { relacionados } & \text { con } \\
\text { la } & \text { salud } \\
\text { psicológica } & \text { y } \\
\text { emocional. } & \end{array}$ \\
\hline $\begin{array}{l}\text { Rospenda et al., } \\
2005\end{array}$ & $\begin{array}{l}\text { Socioculturales } \\
\text { y económicos }\end{array}$ & Acoso sexual & $\begin{array}{l}\text { Sociocultural } \\
\text { Discriminatorio }\end{array}$ & $\begin{array}{l}\text { Problemas en la } \\
\text { salud sexual } \\
\text { (Ansiedad, } \\
\text { depresión, } \\
\text { trastorno de estrés } \\
\text { postraumático } \\
\text { etc.), demandas de } \\
\text { indemnización }\end{array}$ \\
\hline
\end{tabular}

Los estudios tienen como principal limitación la naturaleza ambigua del término mobbing, por ende, un resultado indeseado es que las investigaciones no identifiquen con claridad los factores 
asociados al fenómeno, así mismo la definición está sujeta a la contextualización del ambiente en el que se presenta (véase Tabla 5).

Por otra parte, las investigaciones reconocen a la pertinencia del estudio y verificación de las relaciones causales del fenómeno como un tema de investigación futura. Se plantea la aplicación de estudios con variación geográfica.

Tabla 5.

Hallazgos de los estudios analizados

\begin{tabular}{|c|c|c|c|}
\hline $\begin{array}{c}\text { Autor(es) y } \\
\text { Año }\end{array}$ & Hallazgos & Limitaciones & $\begin{array}{c}\text { Futuras líneas de } \\
\text { investigación }\end{array}$ \\
\hline $\begin{array}{l}\text { Marsh et al., } \\
2009\end{array}$ & $\begin{array}{l}\text { E1 acoso en el lugar de trabajo } \\
\text { es positivamente correlacional } \\
\text { con sintomas de depresión }\end{array}$ & $\begin{array}{l}\text { No habla de factores } \\
\text { protectores o de } \\
\text { técnicas de } \\
\text { investigación }\end{array}$ & $\begin{array}{l}\text { Especificar los } \\
\text { factores protectores y } \\
\text { técnicas de } \\
\text { intervención }\end{array}$ \\
\hline $\begin{array}{l}\text { Coskuner et } \\
\text { al., } 2017\end{array}$ & $\begin{array}{l}\text { Relación positiva y moderada } \\
\text { entre los indicadores } \\
\text { organizacionales y el acoso } \\
\text { laboral }\end{array}$ & $\begin{array}{l}\text { No se presenta una } \\
\text { relación clara en las } \\
\text { variables }\end{array}$ & $\begin{array}{l}\text { Utilizar los mismos } \\
\text { parámetros de la } \\
\text { investigación en } \\
\text { diferentes zonas }\end{array}$ \\
\hline $\begin{array}{l}\text { Merilainen, } \\
\text { Sinkkonen, Pu } \\
\text { hakka, } \\
\text { y Kayhko, } \\
2016\end{array}$ & $\begin{array}{l}\text { Las conductas inapropiadas } \\
\text { Influyen principalmente a } \\
\text { nivel individual como } \\
\text { trastornos afectivos y como } \\
\text { disminución de la } \\
\text { autoconfianza profesional y el } \\
\text { desempeño laboral }\end{array}$ & $\begin{array}{l}\text { E1 estudio reconoce } \\
\text { los roles dentro de } \\
\text { una situación de } \\
\text { acoso, sin embargo, } \\
\text { no habla de sus } \\
\text { orígenes (génesis) }\end{array}$ & $\begin{array}{l}\text { Identificar la relación } \\
\text { causal entre el acoso } \\
\text { laboral y las } \\
\text { relaciones }\end{array}$ \\
\hline
\end{tabular}

\begin{tabular}{|c|c|c|c|}
\hline $\begin{array}{l}\text { Skinner et al., } \\
2015\end{array}$ & $\begin{array}{l}\text { La cultura organizacional es } \\
\text { importante para comprender } \\
\text { el acoso y la intimidación } \\
\text { laboral }\end{array}$ & $\begin{array}{l}\text { Hace un resumen de } \\
\text { la literatura, esto } \\
\text { obstaculiza la } \\
\text { percepción de los } \\
\text { conceptos }\end{array}$ & $\begin{array}{l}\text { Determinar los } \\
\text { comportamientos de } \\
\text { acoso y su génesis }\end{array}$ \\
\hline $\begin{array}{l}\text { Kang y Sidhu, } \\
2015\end{array}$ & $\begin{array}{l}\text { "los incidentes de acoso } \\
\text { sexual en el lugar de trabajo } \\
\text { están en aumento" }\end{array}$ & $\begin{array}{l}\text { Solamente considera } \\
\text { el acoso sexual en el } \\
\text { trabajo }\end{array}$ & $\begin{array}{l}\text { Aplicación en la } \\
\text { cultura mexicana para } \\
\text { posible contrastación } \\
\text { de resultados }\end{array}$ \\
\hline $\begin{array}{l}\text { Moreno, } \\
\text { Sepúlveda y } \\
\text { Restrepo, } \\
2012\end{array}$ & $\begin{array}{l}\text { Los resultados permiten } \\
\text { fundamentar } \\
\text { la necesidad de realizar } \\
\text { intervenciones desde el punto } \\
\text { de vista administrativo y de } \\
\text { bienestar universitario }\end{array}$ & $\begin{array}{l}\text { E1 tamaño del } \\
\text { inventario usado para } \\
\text { medir acoso es } \\
\text { limitado para } \\
\text { establecer una } \\
\text { relación }\end{array}$ & $\begin{array}{l}\text { Investigar la } \\
\text { existencia del acoso } \\
\text { sexual y laboral hacia } \\
\text { los hombres }\end{array}$ \\
\hline $\begin{array}{l}\text { López- } \\
\text { Cabarcos et } \\
\text { al., } 2008\end{array}$ & $\begin{array}{l}\text { La estrategia } \\
\text { más empleada para } \\
\text { enfrentarse al mobbing es la } \\
\text { pasiva, es decir, no hacer } \\
\text { nada }\end{array}$ & $\begin{array}{l}\text { Universo de estudio } \\
\text { muy amplio y } \\
\text { aspectos analizados } \\
\text { solo hacia las } \\
\text { victimas }\end{array}$ & $\begin{array}{l}\text { Analizar aspectos del } \\
\text { perpetrador y } \\
\text { comparar con la } \\
\text { victima }\end{array}$ \\
\hline $\begin{array}{l}\text { Rospenda et } \\
\text { al., } 2005\end{array}$ & $\begin{array}{l}\text { E1 acoso laboral aumenta el } \\
\text { riesgo de enfermedad, lesión } \\
\text { o agresión }\end{array}$ & $\begin{array}{l}\text { Todas las encuestas } \\
\text { daban por resultado } \\
\text { un autoinforme }\end{array}$ & $\begin{array}{l}\text { Desarrollo de medidas } \\
\text { de resultados } \\
\text { establecer la relación } \\
\text { de acoso y las lesiones } \\
\text { (directas, indirectas e } \\
\text { interpersonales) }\end{array}$ \\
\hline
\end{tabular}


En todos los estudios se aseguró la confidencialidad y anonimato de los datos proporcionados por parte de los participantes, así mismo no se ofreció ninguna compensación de ningún tipo, la muestra fue seleccionada de manera aleatoria y representativa. La interacción con los participantes fue de manera directa en el $87.5 \%$ de los estudios analizados, mientras que el resto fue por medio de una plataforma digital. La población se involucró de manera voluntaria y con conocimiento de su participación en la recolección de información, así como su uso.

\section{Discusión}

La revisión sistemática realizada en este trabajo reveló que la cantidad de estudios realizados sobre el tema del acoso laboral en trabajadores universitarios aún sigue siendo limitada, considerando el amplio rango de años que se utilizó para la realización del presente trabajo, aun así, se distingue un alza en la producción científica de este tema durante los últimos 5 años. En lo que se refiere a la distribución geográfica, se infiere una heterogeneidad con lo que respecta al país de realización.

Con relación a los instrumentos, todos ellos han sido utilizados con una diferencia mayor a 5 años con lo que respecta a su fecha de publicación, por lo que ya no contemplan, de manera teórica, las condiciones sociales actuales, además que existe la dificultad para definir el concepto del "mobbing", sus manifestaciones y factores asociados. Esto debido a que es un fenómeno contextual y multifactorial.

Con relación a la metodología empleada, los artículos son de alcance descriptivo y correlacional, esto puede explicarse por la naturaleza del fenómeno, debido a la existencia de distintos factores de riesgo y protección en relación a la incidencia del fenómeno.

De igual forma, la investigación correspondiente al tema del acoso laboral puede continuar bajo la descripción de los factores mencionados, así como la posible relación causal que sostiene con otros factores, como se ha demostrado en diferentes investigaciones previas (Henning, et al., 2017).

Cabe señalar que en la mitad de los trabajos se hallaron incidencias del acoso laboral en el personal universitario superior al 30\%, en la otra mitad se mencionan incidencias menores al 20\%, sin embargo, los mismos estudios asumen que esto se puede deber a múltiples causas, tales como que el tema es definido de diferentes formas en relación con el contexto en el que se presenta.

No obstante, se asume que las manifestaciones más frecuentes son de carácter sociocultural y, en menor medida, sexual; esto en relación con la constante mención de la incidencia de agresiones sexuales y sus consecuencias. Ya que cada estudio analizado identifica diferentes acciones para referirse al acoso laboral y se centra en el motivo causal de éstas. Aunado a esto el análisis por diferencias de género y las conductas de acoso podría aportar con mayor exactitud información necesaria para saber cómo abordar esta problemática, ante los tipos de conductas de acoso y jerarquía del perpetrador o victimario. Sin embargo, $75 \%$ de los estudios incluidos en el análisis no consideran este factor; en todas las investigaciones se identifica al acoso como toda acción cuya motivación sea la de dañar la integridad física, moral y/o psicológica de la víctima.

Añadido a ello, es pertinente señalar que no hay una mención específica dentro de los artículos sobre las consideraciones éticas aplicadas en sus respectivas investigaciones; sumado a ello hay cuestiones metodológicas poco claras como la obtención de la muestra, el periodo de recolección, el procedimiento o el tipo de estudio; es decir, si el estudio es de tipo piloto, es parte de un estudio más grande o una réplica de un estudio anterior, por lo que se sugiere especificar la mención de éstos aspectos para facilitar la réplica del estudio. 
En relación con el contenido de los trabajos analizados, se percibe una ambigüedad conceptual lo que a su vez dificulta la delimitación del fenómeno y, por consecuencia, su estudio en cuanto a factores asociados y sus consecuencias.

En resumen, la propuesta del presente trabajo es la promoción de más estudios sobre el acoso laboral en un ambiente universitario por parte de investigadores latinoamericanos. Así mismo, es preciso establecer una definición correspondiente al acoso laboral, ya que actualmente ésta se encuentra apegada a la contextualización del ambiente sociocultural en el que se presenta. Además de la publicación de los trabajos en idioma inglés para una mayor accesibilidad por parte de los lectores, así como la creación o actualización de instrumentos que permitan la identificación del mobbing. Así mismo, se recomienda la inclusión de las características socioculturales actuales en la formulación de dichos instrumentos.

Una de las limitaciones de la presente revisión sistemática fue la selección de textos completos en español o inglés, ya que pudiese haber artículos cuyo resumen en inglés indique el cumplimiento con las características de selección para análisis, para ello se propone una selección con base en el resumen en inglés y la traducción del contenido por medio de algún recurso físico o electrónico.

\section{Referencias}

Anjum, A., Ming, X., Siddiqi, A., \& Rasool, S. (2018). An Empirical Study Analyzing Job Productivity in Toxic Workplace Environments. International Journal of Environmental Research and Public Health, 15(35), 1-15. Doi: http://dx.doi.org/10.3390/ijerph15051035

Ansoleaga, E., Gómez-Rubio, C., \& Mauro, A. (2015). Violencia laboral en América Latina: una revisión de la evidencia científica. Revista Argentina de Psiquiatría, 26(124), 44-452. Recuperado de http://www.editorialpolemos.com.ar/docs/vertex/vertex124.pdf\#page $=45$

Asquith, N., Ferfolia, T., Brady, B., \& Hanckel, B. (2018). Diversity and safety on campus @ Western: Heterosexism and cissexism in higher education. International Review of Victimology, 3(24), 1-22. doi: https://doi.org/10.1177/0269758018799032

Balducci, C., Cecchin, M., \& Fraccaroli, F. (2012). The impact of role stressors on workplace bullying in both victims and perpetrators, controlling for personal vulnerability factor: a longitudinal analysis. Work \& Stress, 26(3), 195-212. doi: https://doi.org/10.1080/02678373.2012.714543

Barrios, S., \& Paravic, T. (2006). Promoción de la salud y un entorno laboral saludable. La Revista Latino-Americana de Enfermagem, 14(1), 136-141. Recuperado de http://www.scielo.br/pdf/rlae/v14n1/v14n1a19

Bheenuck, S., Miers, M. \& Pollard, K. (2007). Race equality education: Implications of an Audit of student learning. Nurse Education Today, 27(5), 396-405. doi. https://doi.org/10.1016/j.nedt.2006.06.003

Bowling, N., \& Beehr, T. (2006). Workplace harassment from the victim's perspective: BA theoretical model and meta-analysis. Journal of Applied Psychology, 91(5), 998. https://doi.org/10.1037/0021-9010.91.5.998

Coskuner, S., Costur, R., Bayhan-Karapinar, P., Metin-Camgoz, S., Ceylan, S., Demirtas-Zorbaz, S., Aktas, E., \& Ciffiliz, G. (2017). Mobbing, Organizational Identification, and Perceived Support: Evidence from a Higher Education Institution. Eurasian Journal of Educational Research, 73, 19-40. Recuperado de https://dergipark.org.tr/tr/pub/ejer/issue/42503/512026

Eaton, D. (2004). Beyond Room Service: Legal Consequences of Sexual Harassment of Staff by Hotel Guests. Cornell Hospitality Quarterly, 45(4), 347-361. doi: https://doi.org/10.1177/0010880404270064

Einarsen, S., \& Hauge, L. (2006). Antecedentes y consecuencias del acoso psicológico en el trabajo: una revisión de la literatura. Revista de Psicología del Trabajo y de la Organizaciones, 22(3), 251-273. 
Einarsen, S., Hoel, H., Zapf, D., \& Cooper, C. (2003). The concept of bullying at work: the European tradition. En S. Einarsen, H. Hoel, D. Zapf, \& C. Cooper (Eds.), Bullying and Emotional Abuse in the Workplace (pp.3-30). London: Taylor \& Francis.

Fundación Europea para la Mejora de las Condiciones de Vida y Trabajo. (2006). Cuarta encuesta europea sobre las condiciones de trabajo. Irlanda: Oficina de publicaciones europa. Recuperado de http://www.asajaandalucia.es/prevencion/encuestas/encuesta_9/EncuestaEuropeaCondicionesTrabajo.pdf

Gómez, C. (2009). El estrés laboral: una realidad actual. Summa Humanitatis, 3(1), 1-7. Recuperado http://revistas.pucp.edu.pe/index.php/summa_humanitatis/article/view/2330

Hernández, R., Fernández, C., \& Baptista, L. (2014). Metodología de la investigación (6 ${ }^{a}$ edición). Ciudad de México: Mc Graw Hill.

Henning, M. A., Zhou, C., Adamas, P., Moir, F., Hobson, J., Hallett, C., \& Webster, V. S. (2017). Workplace harassment among staff in higher education: a systematic review. Asia Pacific Education Review, 18(4), 521-539. doi: https://doi.org/10.1007/s12564-017-9499-0

Hutton, B., Catalá-López, F., \& Moher, D. (2016). La extensión de la declaración PRISMA para revisiones sistemáticas que incorporan metaanálisis en red: PRISMA-NMA. Medicina Clínica, 147(6), 262-266. doi: https://doi.org/10.1016/j.medcli.2016.02.025

Kang, L. \& Sidhu, H. (2015). Identification of Stressors at Work: A Study of University Teachers in India. Global Business Review, 16(2), 303-320.

López-Cabarcos, M., Picón-Prado, E., \& Vázquez-Rodríguez, P. (2008). Estudio del acoso psicológico en la universidad pública de Galicia. Revista de Psicología del Trabajo y de las Organizaciones, 24(1), 41-60.

Marsh, J., Patel, S., Gelaye, B., Goshu, M., Worku, A., Williams, M. A., \& Berhane, Y. (2009). Prevalence of Workplace Abuse and Sexual Harassment among Female Faculty and Staff. Journal of Occupational Health. Journal of Occupational Health, 51(4), 314-322. Recuperado de https://ci.nii.ac.jp/naid/10025965704/

Merilainen, M., Sinkkonen, H., Puhakka, H., \& Kayhko, K. (2016). Bullying and inappropriate behaviour among faculty personnel. Policy Futures in Education,14(6), 617-634. doi: https://doi.org/10.1177/1478210316639417

Mihyun, P., Sung-Hyun, C., \& Hyun-ja, H. (2014). Prevalence and perpetrators of workplace violence by nursing unit and the relationship between violence and the perceived work environment. Journal of Nursing Scholarship, 47(1), 87-97. doi: https://doi.org/10.1111/jnu.12112

Moreno, L., Sepúlveda, L., \& Restrepo, L. (2012). Discriminación y Violencia de Género en la Universidad de Caldas. Hacia la Promoción de la Salud, 17(1), 59-76.

Nares, M. L., García, P. C., Arvizu, B. A., \& Olimón, A. Y. (2014). Las relaciones humanas en la universidad y el impacto en la calidad educativa. Revista Iberoamericana de Producción Académica y Gestión Educativa, 1(1), 1-20. Recuperado de http://pag.org.mx/index.php/PAG/article/view/62

O’Hare, E., \& O’Donohue, W. (1998). Sexual Harassment: Identifying Risk Factors. Archives of Sexual Behavior, 27(6), 561- 581. doi: https://doi.org/10.1023/A:1018769016832

Ohse, D. M. \& Stockdale, M. S. (2008). Age Comparisons in Workplace Sexual Harassment Perceptions. Sex Roles, 59(3), 240-253. doi: https://doi.org/10.1007/s11199-008-9438-y

Pando, M., Aranda, C., \& Olivares, M. (2012). Análisis factorial confirmatorio del inventario de Violencia y Acoso Psicológico en el Trabajo (IVAPT-PANDO) para Bolivia y Ecuador. Liberabit, 18(1), 27-36.

Parra, L. \& Acosta, M. (2010). La investigación cuantitativa del acoso psicológico laboral en los sectores de la educación superior y de la salud, una revisión sistemática. Entramado, 6(1), 158-172. Recuperado https://revistas.unilibre.edu.co/index.php/entramado/article/view/3367

Piñuel, I. (2001). Mobbing: cómo sobrevivir al acoso psicológico en el trabajo. Madrid: Sal Térrea. 
Romero-Pérez, J. (2006). Mobbing laboral: Acoso moral, psicológico. Revista de Ciencias Jurídicas, 111, 131-162.

Recuperado

de https://revistas.ucr.ac.cr/index.php/juridicas/article/view/9733

Rospenda, K., Richman, J., Ehmke, J. \& Zlatoper, K. (2005). Is workplace harassment hazardous to your health? Journal of Business and Psychology, 20(1), 95-110. doi https://doi.org/10.1007/s10869-005-6992-y

Skinner, T.; Peetz, D.; Strachan, G.; Whitehouse, G.; Bailey, J. \& Broadbent, K. (2015). Selfreported harassment and bullying in Australian universities: explaining differences between regional, metropolitan and elite institutions. Journal of Higher Education Policy and Management, 37(5), 558-571. doi: https://doi.org/10.1080/1360080X.2015.1079400

Tomei, M. (2018). Una experta de la OIT responde a varias preguntas sobre violencia y acoso en el trabajo. Trabajo, (1), 23-24.

Tonon, G. (2012). Las relaciones universidad-comunidad: un espacio de reconfiguración de lo público. Polis, Revista Latinoamericana, 32(12), 1-9.

Trujillo, M., Valderrabano, M. \& Hernández, R. (2007). Mobbing: historia, causas, efectos y propuesta de un modelo para las organizaciones mexicanas. Revista de Ciencias Administrativas y Sociales,17(29), 71-91. Recuperado de https://revistas.unal.edu.co/index.php/innovar/article/view/19586. 